\title{
Uso problemático de Internet y problemas psicológicos entre estudiantes universitarios con discapacidad
}

\section{Problematic Internet Use and Psychological Problems among University Students with Disabilities}

\author{
María J. Pino Osuna *, Carlos Herruzo Pino*, Valentina lucena Jurado*, Javier Herruzo \\ CABRERA*.
}

*Departamento de Psicología. Universidad de Córdoba.

\section{Resumen}

El uso problemático de internet (UPI) entre las personas con discapacidad ha recibido muy poca atención en la literatura científica. El objetivo de este trabajo es estudiar el UPI entre los estudiantes universitarios españoles con discapacidad, y si se relaciona con malestar y problemas psicológicos. En el estudio participaron 432 universitarios españoles con discapacidad de seis universidades (35\%: discapacidad motora, 22,7 \%: discapacidad sensorial y 42,1 \%: otras discapacidades, excluyendo la discapacidad psíquica o intelectual). El UPI se evaluó mediante el Test de Adicción a Internet (IAT), mientras que los problemas psicológicos se evaluaron mediante el cuestionario Clinical Outcomes in Routine Evaluation-Outcome Measure (CORE-OM). La prevalencia del UPI fue del 6,3\%, y no hubo diferencias estadísticamente significativas por sexo, tipo de discapacidad o estudios. Sí hubo diferencias en función de la edad ( $<22$ mostraron una prevalencia 5 veces mayor), origen de la discapacidad (discapacidad congénita mostraron una prevalencia 3,7 veces mayor) y tipo principal de uso (recreativo). La prevalencia de problemas psicológicos es significativamente mayor entre los usuarios problemáticos de Internet, en todas las dimensiones medidas por el CORE-OM (problemas psicológicos, bienestar subjetivo, funcionamiento general y riesgo de autolesión y de daño a otros). En conclusión, los estudiantes universitarios con discapacidad desde el nacimiento (independientemente del tipo de discapacidad y del sexo), menores de 22 años y que utilizan Internet principalmente con fines recreativos y las redes sociales tienen mayor riesgo de sufrir trastornos psicológicos y mayor vulnerabilidad a problemas como ansiedad y dificultades en las relaciones sociales.

Palabras clave: estudiantes con discapacidad, adicción a Internet, problemas psicológicos, funcionamiento, bienestar.

\begin{abstract}
Problematic internet use (PIU) among people with disabilities has received very little attention in scientific literature. The objective of this work is to study PIU among Spanish university students with disabilities, and determine whether it is related to the presence of psychological problems and discomfort. A total of 432 Spanish university students with disabilities from six universities participated in the study (35\% motor disability, $22.7 \%$ sensory disability and $42.1 \%$ other disabilities, excluding psychical or intellectual disability). PIU in the sample was assessed using the Internet Addiction Test (IAT), while psychological problems were assessed using the Clinical Outcomes in Routine Evaluation-Outcome Measure (CORE-OM). PIU prevalence was $6.3 \%$, and significant differences were found by age $(<22$ had a PIU prevalence five times higher), onset of disability (prevalence was 3.7 times higher in those with congenital disability) and main type of internet use (recreational). There were no differences by sex, level of education or type of disability. In all the dimensions measured by CORE-OM (psychological problems, subjective wellbeing, life functioning and risk of self-harm and harm to others), the prevalence of psychological problems was significantly higher among problematic internet users. In conclusion, university students who are disabled from birth (regardless of their type of disability and sex), under the age of 22, and use the internet mainly for social networking and recreational purposes are at greater risk of psychological distress and are more vulnerable to problems like anxiety and difficulties with social relationships.

Key words: students with disabilities, internet addiction, psychological problems, life functioning, wellness.
\end{abstract}


D urante las últimas tres décadas, el uso de internet se ha disparado. Hoy representa tal porcentaje de las actividades cotidianas que está modificando de manera significativa las dinámicas de las relaciones sociales, formas de comunicación, movilidad, etc. (Hernández, Ortiz y Uribe, 2013). Sin embargo, en paralelo a este aumento del uso de las nuevas tecnologías, es cada vez mayor la preocupación respecto de dónde exactamente yacen los límites entre el uso adecuado e inadecuado de las mismas. Han surgido en la literatura científica varios términos diferentes para referirse a este fenómeno. Estos incluyen «adicción a Internet», «uso patológico de Internet», «uso problemático de Internet» (UPI) y «uso excesivo de Internet» (Echeburúa y Corral, 2010); todos se refieren a una incapacidad para controlar el uso de Internet que resulta en dificultades psicológicas y sociales y que afectan los estudios y el trabajo en la vida personal (Spada, 2014; Zhou, Li, Li, Wang y Zhao, 2017). Sin embargo, la existencia misma de adicción a Internet es hoy todavía un tema de debate. La clasificación en el DSM-5 (American Psychiatric Association, 2013), a pesar de reconocer trastornos adictivos no relacionados con sustancias dentro de su categoría de Trastornos adictivos relacionados con las sustancias, solo especifica el trastorno de juego patológico y deja otras adicciones conductuales como fenómenos a ser estudiados en futuras investigaciones. La CIE-11, que incluye los trastornos de juegos de azar en línea y videojuegos, tiene el mismo enfoque (OMS, 2019).

Al mismo tiempo, el tema ha atraído una atención creciente por parte de la comunidad científica a lo largo de este periodo, con un aumento exponencial en el número de publicaciones dedicadas a la adicción a Internet o UPI (Kuss, Griffiths y Binder, 2013; Lucena, Raya-Trenas, Herruzo y Herruzo, 2019; Ruiz-Olivares, Lucena, Pino y Herruzo, 2010). Esto se refleja en la profusión de instrumentos que han surgido para evaluar cómo se usan las diferentes tecnologías (teléfonos móviles, Internet, redes sociales, videojuegos, etc.), más de 20 de los cuales se centran específicamente en el uso de Internet (Kraus y Rosenberg, 2014).

A pesar de este aumento general de la investigación del uso normal tanto como del uso problemático de las tecnologías (Carbonell, Fúster, Chamorro y Obsert, 2012), la literatura ha dedicado muy poca atención a determinados grupos de personas que están particularmente afectados por los cambios en los hábitos de vida provocados por este tipo de tecnologías. Uno de estos grupos es el de las personas con discapacidad (Suriá, 2012a). Las características específicas de estas personas y sus condiciones de vida, que en algunos casos las ponen en riesgo de aislamiento social, hacen necesario examinar cómo este grupo vive el gran cambio social de la revolución de las TIC y determinar si les está afectando de forma negativa el uso y abuso de las tecnologías en mayor o menor medida que la población «no discapacitada» (Duplaga y Sluzc, 2019). Las nuevas tecnologías, sin duda, han ayudado a las personas con discapacidad a obtener mayor autonomía e independencia (Igual, Plaza, Medrano y Rubio, 2014). Los teléfonos móviles, por ejemplo, facilitan el contacto con personas que no están en el mismo espacio físico, tanto para personas con y sin discapacidad (Campbell y Ling, 2009; Perry, O’Hara, Sellen, Brown y Harper, 2001), y pueden ser útiles para eliminar algunas de las barreras que enfrentan las personas con movilidad reducida gracias a aplicaciones que promueven la autonomía, facilitan las tareas diarias, y, por tanto, disminuyen el sentido de estigmatización asociada a tener una discapacidad (Chib y Jiang, 2014). De manera coherente, Söderström (2009) sostiene que el uso de los teléfonos inteligentes ha facilitado la construcción de nuevas identidades en las personas con discapacidad física, permitiéndoles «esconderse» y así aumentar su percepción del empoderamiento, ampliar sus relaciones sociales y ayudándoles a expandir su zona de confort. Söderström también informa de que las personas con discapacidad valoran el teléfono móvil como una herramienta esencial de comunicación en un grado mayor que las personas sin discapacidad, dado que les permiten solicitar ayuda en determinadas situaciones cotidianas que las personas sin discapacidad probablemente no experimentan con la misma frecuencia (Söderström, 2011).

En la literatura, sin embargo, se ha prestado muy poca atención a los problemas relacionados con el uso de Internet experimentados por las personas con discapacidad. Los estudios se han centrado casi exclusivamente en la discapacidad intelectual o el fenómeno de ciberacoso (Simpson, Rose y Ellis, 2016) que sufren personas con discapacidad de distintas edades (Heiman, Olenik-Shemesh y Eden, 2015; Jenaro, et al., 2018), siendo el acoso mayor cuanto más «visible» es la discapacidad (Kowalski, Morgan, Drake-Lavelle y Allison, 2016). En el caso del ciberacoso, las TIC son un medio a través del cual ocurre la conducta violenta, pero la literatura que aborda la cuestión del uso normal o problemático de las tecnologías por parte de las personas con discapacidad, independientemente de los posibles casos de adicción, es escasa. Lathouwers, de Moor y Didden (2009) estudiaron la forma en que 97 adolescentes con discapacidad física acceden y utilizan Internet en los Países Bajos. No hallaron diferencia alguna en cómo los adolescentes con y sin discapacidad física acceden al Internet, y las actividades en línea más comunes fueron similares para ambos grupos, aunque el abuso no se ha estudiado en profundidad. Además, Suriá (2015) comparó el uso abusivo de Internet en una muestra de jóvenes con y sin discapacidad y encontró un mayor nivel de abuso de las tecnologías entre personas con discapacidad motora que entre personas sin discapacidad (Suriá, 2012b), mostrando ambos grupos niveles similares de uso, alrededor del $90 \%$, durante la adolescencia (Adecco, 2017). La muestra utilizada en el estudio de Suriá, sin embargo, fue pequeña y el análisis no abordó cómo los resultados se relacionaron con los problemas psicológicos 
encontrados en personas sin discapacidad (Spada, 2014; Zhou et al., 2017). Como ya han destacado varias revisiones críticas (Aponte, Castillo y González, 2017; James y Tunney, 2017), el factor más relevante desde el punto de vista social es el efecto sobre el bienestar de las personas.

Por tanto, dado que todas las personas, incluso aquellas con algún tipo de discapacidad, se exponen a los efectos tanto beneficiosos como perjudiciales del uso de Internet, y en vista de la escasez de estudios realizados sobre el UPI entre personas con discapacidad, el objetivo de este trabajo fue estudiar el UPI entre universitarios españoles con discapacidad, y determinar si está relacionada con la presencia de problemas psicológicos y malestar.

\section{Método}

\section{Participantes}

Un total de 432 universitarios españoles con discapacidad participó en el estudio. El $35 \%$ tenía una discapacidad motora, el 22,7 \% una discapacidad sensorial, y el 42,1\% otras discapacidades (excluyendo discapacidad cognitiva). El $25 \%$ tenía una discapacidad congénita, mientras que el $75 \%$ la tenía adquirida. El 54,4\% de la muestra era mujer y el 45,6 \% era hombre. La edad media fue de 41,90 años ( $S D$ $=13,77)$. La homocedasticidad se verificó usando la prueba de chi cuadrado, comparando los grupos según tipo de discapacidad y sexo. Dada la ausencia de resultados significativos, se puede decir que la muestra fue homogénea en estos dos aspectos $\left(\chi^{2}=2,70 ; p=, 257\right)$. Los participantes fueron reclutados a través de una invitación enviada por correo electrónico por parte de los Servicios de Atención a Universitarios con Discapacidad (UNED [Universidad Nacional de Educación a Distancia] y las universidades de Valencia, Cádiz, Málaga, Jaén, La Laguna, Barcelona y País Vasco) a los estudiantes registrados en sus bases de datos, junto con un enlace a una página web. De un total de 560 personas que accedieron al sitio web, el $23 \%$ rehusó responder a la encuesta. Con respecto a las áreas de estudio, el 24,4 \% eran estudiantes de Humanidades; el 41,4 \% de Ciencias Sociales, Económicas y Jurídicas; el 11,9 \% de Ciencias y Tecnologías y el 22,4 \% de Ciencias de la Salud. Por tanto, en general, el $34,2 \%$ estudiaba ciencias y tecnologías, y el 65,8 \% estudiaba ciencias sociales y humanidades.

\section{Instrumentos}

La primera parte de la encuesta incluyó una serie de preguntas sobre los tipos de discapacidad de los participantes, la naturaleza de la discapacidad (congénita o adquirida) y los años transcurridos desde su inicio. Las preguntas sobre el tipo de discapacidad incluyeron diferentes opciones (auditiva, visual, física y otras). Los estudiantes con discapacidad fueron contactados a través de los servicios de apoyo a los estudiantes con discapacidad de las universidades participantes. Para acceder a dichos servicios, el tipo y grado de discapacidad del estudiante debe haber sido acreditado por una institución sanitaria reconocida.

Se incluyó también un conjunto de preguntas sobre el uso de Internet por parte de los participantes (porcentaje de tiempo en línea dedicado a ocio, trabajo, estudios y redes sociales). Para facilitar las respuestas, las opciones de respuesta se establecieron como porcentajes (0-25 \%, 25$50 \%, 50-75 \%, 75-100 \%$ ). Cuando un participante dio una respuesta $75-100 \%$, se consideró que usaba Internet principalmente para el propósito en cuestión. También se incluyeron preguntas sobre otras características sociodemográficas (edad, sexo, nota media el año anterior, año de estudios, especialización, universidad).

También se aplicaron los siguientes instrumentos:

Prueba de adicción a Internet de Young (1998), adaptada para hispanohablantes por Carbonell et al. (2012) y validada por Fernandez-Villa et al. (2015a) y Pino, Herruzo, Raya, Ruiz-Olivares y Herruzo (2020). Esta prueba analiza el grado en que el uso de Internet afecta a la vida diaria, vida social, productividad, sueño y sentimientos de una persona. Consta de 20 ítems, evaluados en una escala Likert de seis puntos ( 0 = Nunca; 1 = Casi nunca; 2 = Ocasionalmente; 3 = Con frecuencia; 4 = A menudo; $5=$ Siempre). La literatura informa sobre las estructuras internas que varían entre una y seis dimensiones. En este estudio, el Análisis Factorial Exploratorio encontró una estructura tridimensional (CMIN/ $\mathrm{DF}=3,159 ;$ AGFI $=, 95 ; \mathrm{CFI}=, 903 ; \mathrm{NFI}=, 865 ; \mathrm{IFI}=, 904 ;$ TLI $=, 876$; RMSEA [90\% IC] $=, 073[, 066-, 081])$. La autora (Young, 2011) propone una puntuación de 30 como límite entre el uso normal y patológico. Otros investigadores, como Jelenchick, Becker y Moreno (2012) fijan el límite en 40 puntos. Dada la controversia sobre el número de factores existentes, en este trabajo decidimos analizar el UPI en base a un punto de corte de 40 , considerando a quienes obtuvieron puntuaciones de 40 o más como usuarios problemáticos, como han hecho otros estudios con población española (Fernández-Villa et al., 2015a; Fernández-Villa et al. 2015b). El coeficiente de consistencia interna alfa de Cronbach se acerca a ,90. En el presente estudio, el coeficiente alfa de la muestra fue de, 925 .

El Clinical Outcomes in Routine Evaluation-Outcome Measure (CORE-OM), de Evans et al. (2002), adaptado para la población española por Trujillo et al. (2016). Es un cuestionario de autoinforme compuesto por 34 ítems que evalúan el estado del individuo en base a cuatro dimensiones: 1) bienestar/malestar subjetivo (4 ítems); 2) problemas/síntomas (12 ítems, medición de ansiedad, depresión, trauma y síntomas físicos); 3) funcionamiento general (12 ítems, evaluación de relaciones íntimas, relaciones sociales y niveles de funcionamiento diario); y 4) riesgo (4 ítems que sirven como indicadores clínicos de intentos de suicidio y autolesiones, y 2 ítems para predecir actos de agresión contra terceros). Las puntuaciones medias por debajo de 1 indican niveles saludables. Las propiedades psicométricas de 
este test han demostrado niveles aceptables de consistencia interna (valores alfa entre ,75 y ,90) y de sensibilidad en las mediciones obtenidas (Evans et al., 2002) y se ha utilizado en numerosos contextos clínicos (Connell, et al., 2007; Palmieri et al., 2009) y universitarios (Connell, et al., 2007; Botella, 2006).

\section{Procedimiento}

Los procedimientos del estudio se implementaron conforme a la Declaración de Helsinki. Una vez que el estudio fue aprobado por la Junta de Revisión Institucional (Comité de Ética) del gobierno regional de Andalucía (ref. 3050, registro 249), se contactó con los Centros de Atención a Universitarios con Discapacidad. Estos centros remitieron por correo electrónico a sus usuarios una invitación a hacer clic en un enlace para participar en una encuesta sobre el uso de las nuevas tecnologías por parte de estudiantes con discapacidad. En el correo electrónico y en la primera página del cuestionario, se les informó que al completar la encuesta daban su consentimiento a la utilización de sus respuestas exclusivamente para fines de investigación y con total confidencialidad, y que no se registraría ningún dato aparte de sus respuestas a la encuesta. Para obtener el consentimiento informado, el texto también incluía: «Al completar esta encuesta, está de acuerdo en participar voluntariamente en este estudio. Puede negarse a participar en el estudio en cualquier momento sin necesidad de dar explicaciones por ello».

\section{Análisis de datos}

Dado que la estructura del instrumento había sido motivo de controversia y no había sido validado con estudiantes con discapacidad, en primer lugar, se evaluaron sus dimensiones subyacentes. Para este propósito utilizamos la medida de la adecuación muestral de Kaiser-Mayer-Olkin (KMO = ,938), que verificó la idoneidad de la muestra. La prueba de esfericidad de Bartlett x2 = 3434,76; $p<, 001$ indicó una relación significativa entre las variables y la viabilidad de aplicar un análisis factorial exploratorio. Se realizó un aná- lisis de los componentes principales con rotación Varimax y normalización de Kaiser para agrupar los 20 ítems del IAT en tres componentes con autovalores superiores a 1, lo que explica el 47,80 \% de la varianza. Se realizó un análisis factorial confirmatorio (AFC) de la muestra con el programa AMOS.18, y los valores de ajuste fueron aceptables o buenos (Chi2 = 470,753, $p<, 001$; discrepancia mínima dividida por grados de libertad DMIN/DF $=3,159(<5)$; índice de bondad de ajuste ajustado: AGFI $=, 95(>, 9)$; índice de ajuste comparativo CFI = ,903 (> ,9); IFI = ,904 (> ,9); índice de Tucker-Lewis TLI = ,876 (cercano a 1); índice de ajuste de parsimonia: error cuadrático medio de aproximación RMSEA [IC $90 \%]=, 073[, 066-, 081](<, 08))$.

Las prevalencias se calcularon con sus respectivos intervalos de confianza de $95 \%$, mientras que las variables numéricas se analizaron mediante el cálculo de desviaciones medias y estándar. La relación entre el UPI y las dificultades psicológicas, sociales y académicas y otras variables se estudiaron con modelos de regresión logística bivariable y multivariable, calculando las Odds Ratios con sus respectivos intervalos de confianza y ajustando por edad, sexo y rama de estudios. Estos análisis se realizaron utilizando el paquete estadístico SPSS.

\section{Resultados}

Como se indica en el apartado de Análisis, primero se realizó un análisis factorial exploratorio, seguido de un análisis confirmatorio para comprobar la estructura factorial y el ajuste del cuestionario, el cual no se había utilizado antes con personas con discapacidad. Dada la controversia respecto de su estructura, sin embargo, en el presente trabajo se utilizó la puntuación del cuestionario completo, con un punto de corte para UPI de 40, tal como se menciona en el apartado de Instrumentos.

Como muestra la Tabla 1, la prevalencia de UPI en la muestra fue del 6,3\%, sin diferencias estadísticamente significativas por sexo y tipo de discapacidad. Con respecto a la edad, los participantes menores de 22 años tuvieron una

Tabla 1. Prevalencia de UPI por variables sociodemográficos.

\begin{tabular}{|c|c|c|c|c|c|c|c|c|}
\hline & & $\mathbf{N}$ & $\mathbf{n}$ & $\%$ & 0 & $95 \%$ IC & B & $p$ \\
\hline \multirow[t]{2}{*}{ Tipo de discapacidad } & Motora & 142 & 9 & 6,3 & 1,203 & ,464-3,117 & ,185 & ,704 \\
\hline & Sensorial & 87 & 6 & 6,9 & 1,317 & $, 453-3,828$ &, 275 & ,613 \\
\hline \multirow[t]{2}{*}{ Edad } & $<22$ & 41 & 8 & 19,5 & 1 & & & \\
\hline & $>=22$ & 357 & 16 & 4,5 & ,194 & ,077-,486 & $-1,642$ & $<, 001$ \\
\hline Origen & Congénita & 90 & 11 & 12,2 & 1 & & & \\
\hline \multirow[t]{2}{*}{$\overline{\text { Sexo }}$} & Hombre & 181 & 12 & 6,6 & 1 & & & \\
\hline & Mujer & 217 & 12 & 5,5 & 1,213 & $, 531-2,770$ & ,193 &, 647 \\
\hline \multirow[t]{2}{*}{ Estudios } & Ciencias/Tecnología & 124 & 8 & 6,5 & 1 & & & \\
\hline & Humanidades/Ciencias Sociales/Económicas/Derecho & 238 & 14 & 5,9 & ,906 & ,370-2,223 &,- 098 & ,830 \\
\hline
\end{tabular}

Nota. N: Usuarios no problemáticos de Internet. n: Usuarios problemáticos. \%: Porcentaje de usuarios problemáticos. OR: Odds Ratio. B: Coeficiente B. 
prevalencia (Odds Ratio: OR) cinco veces mayor que los estudiantes más mayores. En los participantes con discapacidad congénita, la prevalencia de UPI fue 3,7 veces superior.

Como muestra la Tabla 2, la prevalencia de puntuaciones en CORE-OM por encima del punto de corte de riesgo de problemas clínicos (malestar clínicamente significativo) era significativamente mayor entre los estudiantes con UPI en todas las dimensiones medidas por este instrumento, que entre aquellos con valores normales, con valores de Odds Ratio entre 3,18 para relaciones íntimas hasta 6,8 para relaciones sociales, y desde 5,39 para bienestar subjetiva hasta 5,37 para ansiedad.
También se exploró la relación entre diferentes tipos de uso de Internet (principalmente para el trabajo y los estudios, las redes sociales y para otras actividades recreativas) (ver Tabla 3). El $50 \%$ de los que dedicó más del $75 \%$ de su tiempo en Internet a actividades recreativas tenía UPI, en comparación con solo el 4,9\% de los que pasaron menos tiempo en el uso recreativo. Sin embargo, en el caso de las personas que utilizan el Internet principalmente para el trabajo y los estudios, el resultado se invirtió: el 1,9 \% de los que lo utilizaban más del $75 \%$ del tiempo para estudiar tenía UPI, y el porcentaje de los que lo utilizaban menos del $75 \%$ del tiempo para el trabajo y los estudios, aumentó a 7,5\%.

Tabla 2. Relaciones entre UPI (IAT) y problemas psicológicos (CORE-OM).

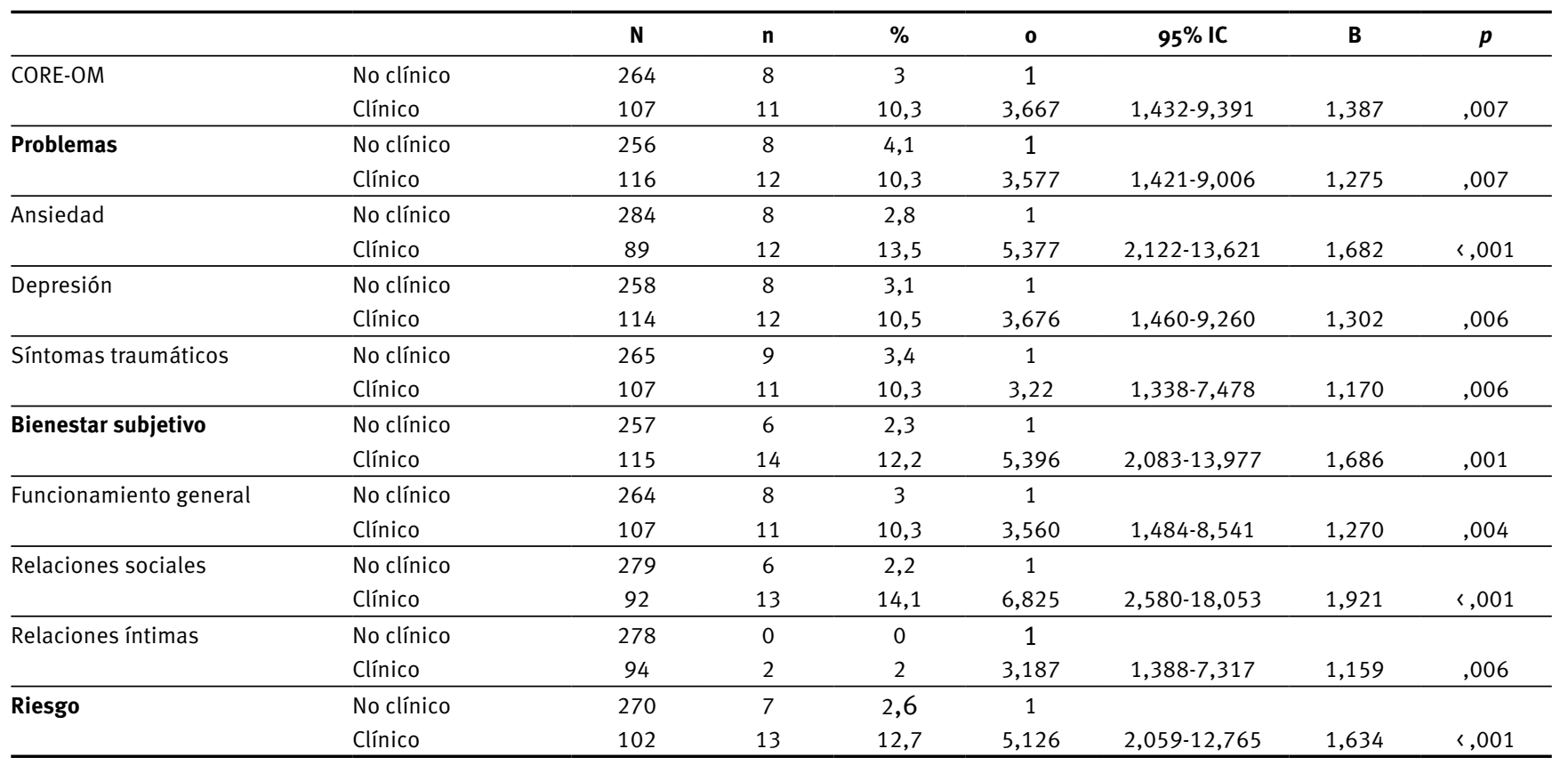

Nota. N: Usuarios no problemáticos de Internet. n: Usuarios problemáticos. \%: Porcentaje de usuarios problemáticos. OR: Odds Ratio. B: Coeficiente B.

Tabla 3. Relaciones entre UPI (IAT) y tipo de uso de Internet.

\begin{tabular}{|c|c|c|c|c|c|c|c|c|}
\hline Tipo de uso & Porcentaje de uso & $\mathbf{N}$ & $\mathbf{n}$ & $\%$ & $\mathbf{0}$ & $95 \%$ IC & B & $p$ \\
\hline \multirow[t]{2}{*}{ Trabajo y estudio } & $0-75 \%$ & 272 & 22 & 7,5 & 1 & & & \\
\hline & $75-100 \%$ & 102 & 2 & 1,9 & ,141 & ,027-, 740 & $-1,959$ & ,021 \\
\hline \multirow[t]{2}{*}{ Redes sociales } & $0-75 \%$ & 351 & 16 & 4,3 & 1 & & & \\
\hline & $75-100 \%$ & 23 & 8 & 25,8 & 7,630 & $2,484-22,651$ & 2,015 & $<, 001$ \\
\hline \multirow[t]{2}{*}{ Recreativo } & $0-75 \%$ & 369 & 19 & 4,9 & 1 & & & \\
\hline & $75-100 \%$ & 5 & 5 & 50 & 10,865 & $2,494-47,335$ & 2,386 & ,001 \\
\hline
\end{tabular}

Nota. N: Usuarios no problemáticos de Internet. n: Usuarios problemáticos. \%: Porcentaje de usuarios problemáticos. OR: Odds Ratio. B: Coeficiente B.

\section{Discusión}

El objetivo de este estudio fue analizar el UPI entre estudiantes universitarios con discapacidad y determinar si estaba relacionado con la presencia de problemas psicológicos y molestias. Los resultados mostraron que los estudiantes ma- yores de 22 años de edad con una discapacidad adquirida y que utilizan Internet principalmente para el trabajo y/o los estudios tienen menor riesgo de UPI, independientemente de su tipo de discapacidad, sexo, o rama de estudios. Por el contrario, tienen un mayor riesgo de UPI los menores de 22 
años con una discapacidad congénita y cuyo uso de Internet es, sobre todo, de redes sociales y otras actividades recreativas. También se encontró que el UPI estaba asociado con mayor prevalencia de problemas psicológicos y malestar.

La tasa de UPI entre las personas con discapacidad resultó ser del 6,3\%, una cifra similar a la obtenida por Fernández-Villa et al. (2015a) utilizando el mismo instrumento en universitarios sin discapacidad. Esto contradice los resultados de Suriá (2015), quien encontró un mayor nivel de uso problemático entre los estudiantes con discapacidad que entre los estudiantes sin discapacidad. Sin embargo, estas diferencias pueden deberse al pequeño tamaño de la muestra de Suriá $(n=128)$ y el hecho de que ella aplicó un instrumento diferente, el «Cuestionario de Experiencias Relacionadas con Internet» (CERI) desarrollado por Beranuy, Chamarro, Graner y Carbonell (2009). Este cuestionario utiliza los criterios del DSM-IV-TR para abuso de sustancias y juego patológico y, al igual que el IAT utilizado en el presente estudio, se ha validado para la población española, por lo que es muy probable que las diferencias halladas se deban al pequeño tamaño muestral y/o el método de muestreo («bola de nieve»).

Nuestros resultados no mostraron diferencias con respecto al tipo de discapacidad. Esto también contradice los resultados obtenidos por Suriá (2015), que mostraron una mayor prevalencia de UPI entre las personas con discapacidad motora. Son necesarios más estudios para ayudar a aclarar estas discrepancias, pero, de nuevo, es probable que se expliquen por el uso de Suriá del método de muestro de «bola de nieve». Es más, nuestro estudio encontró diferencias significativas según el origen de la discapacidad, con una mayor prevalencia entre los estudiantes con una discapacidad congénita que entre los que tienen una discapacidad adquirida. Las cifras que obtuvimos son inevitablemente dinámicas debido a los instrumentos que usamos y porque el UPI es un fenómeno cambiante y probablemente creciente. Este problema claramente afecta a un porcentaje importante de la población con discapacidad, por lo que se necesita más investigación.

Dada la escasez de la literatura sobre el problema de UPI en personas con discapacidad, nuestro estudio es uno de los primeros en haber aportado datos que aclaran el impacto que las nuevas tecnologías están teniendo en este colectivo. Duplaga y Sluzc (2019) encontraron que el uso de Internet estaba relacionado con sentimientos de felicidad y mejor salud mental, pero en su estudio solo incluyeron una pregunta dicotómica sobre si las personas usaban o no Internet. Nuestro estudio, sin embargo, se basa en un instrumento que mide directamente el UPI. Los resultados obtenidos por Duplaga y Sluzc (2019) confirman que el uso no problemático de Internet tiene un efecto positivo en el sentido de que puede facilitar mayor autonomía, independencia (Igual et al., 2014) y contacto con personas que no están en el mismo espacio físico (Campbell y Ling, 2009; Perry et al., 2001) y también puede ser útil para eliminar algunas de las barreras que enfrentan las personas con movilidad reducida (Chib y Jiang, 2014).

Nuestro estudio también muestra que las personas con discapacidad con UPI tienen significativamente más problemas psicológicos que aquellos cuyo uso de dicha tecnología es normal o más controlado. En este sentido, Kraut et al. (1998) informaron de una correlación positiva entre el uso de Internet y la depresión, la soledad y el estrés. Miller (2008) también encontró una relación negativa significativa entre la frecuencia de uso de videojuegos y grados de bienestar en personas con lesiones de la médula espinal. El estudio actual confirma la relación hallada en otros estudios entre el UPI y la presencia de angustia psicológica y problemas como depresión, ansiedad y dificultades con relaciones sociales en la población de personas con discapacidad.

Es de destacar que en todas las dimensiones de angustia psicológica estudiadas con el CORE-OM, un instrumento que proporciona puntos de corte para indicar la presencia de problemas, la prevalencia es significativamente mayor entre aquellos con UPI. Los datos obtenidos en este estudio muestran que las cifras de prevalencia de angustia psicológica y ansiedad son también más de cinco veces más altas. Las cifras obtenidas para el riesgo de autolesión o daño a los demás (Riesgo), que también son 5 veces más altas entre las personas con UPI que entre aquellas cuyo uso de Internet es normal, refuerzan la idea de UPI como un problema real digno de atención, sin ni siquiera entrar en la polémica de si el uso excesivo de Internet debe o no considerarse una adicción.

Nosotros también encontramos que las relaciones sociales problemáticas son más de seis veces más frecuentes entre aquellos con UPI que entre aquellos sin UPI. Teniendo en cuenta los datos obtenidos por Duplaga y Sluzc (2019) y Campbell y Ling (2009), esto parece sugerir que el uso adecuado de Internet favorece la inclusión, pero que el UPI favorece el aislamiento.

En resumen, la relación entre, por una parte, la angustia psicológica, los problemas psicológicos, las malas relaciones sociales y la ansiedad y, por otra parte, el UPI, parece indicar que la «adicción» a Internet puede cumplir una función de evitación del malestar a través del aislamiento social o la evitación experiencial, como ya sugirieron otros estudios (James y Tunney, 2017; Lucena et al., 2019), y que esto puede estar fomentando más aislamiento y, por tanto, más adicción. Esto sería coherente con la mayor prevalencia de UPI hallada entre personas con discapacidad congénita y entre las que utilizan el Internet principalmente para otros fines recreativos. Sin embargo, la naturaleza transversal del estudio nos impide establecer si son los problemas psicológicos los que conducen al UPI o es el UPI el que genera los problemas psicológicos, o si ambos tipos de problema se refuerzan mutuamente, por lo que son necesarios estudios adicionales de diseño longitudinal. 
En conclusión, los universitarios menores de 22 años, con discapacidades congénitas (independientemente del tipo) y que utilizan el Internet principalmente para redes sociales y con fines recreativos tienen mayor riesgo de malestar psicológico y problemas.

\section{Reconocimientos}

Fuentesdefinanciación:ProyectoPID2020?117172RB-100 financiado por FEDER/Ministerio de Ciencia e Innovación - Agencia Estatal de Investigación (España). Los autores desean agradecer a los Servicios de Atención a Universitarios con Discapacidad de la Universidad Nacional de Educación a Distancia (UNED) y las Universidades de Valencia, País Vasco, Jaén, Cádiz, Málaga, La Laguna y Barcelona por su colaboración.

\section{Conflicto de interés}

Los autores declaran la inexistencia de conflicto de interés.

\section{Referencias}

Adecco (2017). Informe Infoempleo- Adecco. Redes Sociales y Mercado de Trabajo en España. Recuperado de https:// www.adeccogroup.es/wp-content/uploads/2017/11/ Informe-2017-Empleo-y-Redes.-Infoempleo-Adecco.pdf.

American Psychiatric Association (2013) Diagnostic and statistical manual of mental disorders (5th ed). Washington, DC: American Psychiatric Association.

Aponte, R., Castillo, P. y González, J. (2017). Prevalencia de adicción a internet y su relación con disfunción familiar en adolescentes. Revista Clinica de Medicina de Familia, 10, 179-186.

Botella, L. (2006). Routine evaluation in psychotherapy service: The use of CORE System data. European Journal of Psychotherapy E Counselling, 8, 235-241. doi:10.1080/13642530600714672.

Beranuy Fargues M., Chamarro Lusar A., Graner Jordania C. y Carbonell Sánchez X. (2009). Validación de dos escalas breves para evaluar la adicción a internet y el abuso de móvil. Psicothema, 21, 480-485.

Campbell, S. W. y Ling, R. (2009). Effects of mobile communication. Media Effects: Advances in Theory and Research, 592-606. Recuperado de http:/ / citeseerx.ist.psu. $\mathrm{edu} / \mathrm{viewdoc} /$ download?doi=10.1.1.501.7868\&rep=rep1\&type $=$ pdf.

Carbonell, X., Fúster, H., Chamarro, A. y Oberst, U. (2012). Internet and mobile phone addiction: A review of Spanish empirical studies. Psychologist's Papers, 33, 82-89.

Chib, A. y Jiang, Q. (2014). Investigating modern-day Talaria: mobile phones and the mobility-impaired in Sin- gapore. Journal of Computer-Mediated Communication, 19, 695-711. doi:10.1111/jcc4.12070.

Connell, J., Barkham, M., Stiles, W. B., Twigg, E., Singleton, N., Evans, C. y Miles, J. V. (2007). Distribution of CORE-M scores in a general population, clinical cut-off points and comparison with the CIS -R. British Journal of Psychiatry, 190, 69-74. doi:10.1192/bjp.bp.105.017657.

Duplaga M. y Szulc K., (2019). The association of internet use with wellbeing, mental health and health behaviours of persons with disabilities. International Journal of Environmental Research and Public Heatlh, 16, 3252. doi:10.3390/ijerph16183252.

Echeburúa, E. y De Corral, P. (2010). Addiction to new technologies and social networks in young people: A new challenge. Addictions, 22, 91-96.

Evans, C., Connell, J., Barkham, M., Margison, F., McGrath, G., Mellor-Clark, J. y Audin, J. (2002). Towards a standardised brief outcome measure: Psychometric properties and utility of the CORE-OM. British Journal of Psychiatry, 180, 51-60. doi:10.1192/bjp.180.1.51.

Fernández-Villa, T. Molina, A., García-Martín, M. Llorca, J., Delgado-Rodríguez, M. y Martín, V. (2015a). Validation and psychometric analysis of the Internet Addiction Test in Spanish among college students. BMC Public Health, 15, 953-963. doi:10.1186/s12889-015-2281-5.

Fernández-Villa, T., Alguacil, J., Almaraz, A., Cancela, J. M., Delgado-Rodríguez, M., García-Martín, M. ... Martín, V. (2015b). Problematic internet use in university students: Associated factors and differences of gender. Adicciones, 27, 265-275. doi:10.20882/adicciones.751.

Heiman, T., Olenik-Shemesh, D. y Eden, S. (2015). Cyberbullying involvement among students with ADHD: Relation to loneliness, self-efficacy and social support. European Journal of Special Needs Education, 30, 15-29.

Hernández, J., Ortiz, R. y Uribe, A. (2013). Innovation and technological knowledge in the society of the 21 st century: The ICT revolution. Nueva Época, 13, 89-96.

Igual, R., Plaza, I., Medrano, C. y Rubio, M. A. (2014). Personalizable smartphone-based system adapted to assist dependent people. Journal of Ambient Intelligence and Smart Environments, 6, 569-593. doi:10.5555/2693820.2693822.

James, R. J. y Tunney, R. J. (2017). The need for a behavioural analysis of behavioural addictions. Clinical Psychology Review, 52, 69-76. doi:10.1016/j.cpr.2016.11.010.

Jelenchick, L. A., Becker, T. y Moreno, M. A. (2012). Assessing the psychometric properties of the Internet Addiction Test (IAT) in US college students. Psychiatry Research, 196, 296-301. doi:10.1016/j.psychres.2011.09.007.

Jenaro, C., Flores, N., Vega, V., Cruz, M., Pérez, M. C. y Torres, V. A. (2018). Cyberbullying among adults with intellectual disabilities: Some preliminary data. Research in Developmental Disabilities, 72, 265-274. doi:10.1016/j. ridd.2017.12.006. 
Kowalski, R. M., Morgan, C. A., Drake-Lavelle, K. y Allison, B. (2016). Cyberbullying among college students with disabilities. Computers in Human Behavior, 57, 416-427. doi:10.1016/j.chb.2015.12.044.

Kraus, S. y Rosenberg, H. (2014). The pornography craving questionnaire: Psychometric properties. Archives of Sexual Behavior, 43, 451-462. doi:10.1007/s10508-0130229-3.

Kraut, R., Patterson, M., Lundmark, V., Kiesler, S., Mukophadhyay, T. y Scherlis, W. (1998). Internet paradox: A social technology that reduces social involvement and psychological well-being? American Psychologist, 53, 1017-103. doi:10.1037/0003-066X.53.9.1017.

Kuss D. J., Griffiths M. D. y Binder J. F. (2013). Internet addiction in students: prevalence and risk factors. Computers in Human Behavior. 29, 959-66. doi:10.1016/j. chb.2012.12.024.

Lathouwers, K., de Moor, J. y Didden, R. (2009). Access to and use of internet by adolescents who have a physical disability: A comparative study. Research in Developmental Disabilities, 30, 702-711. doi:10.1016/j.ridd.2008.09.003.

Lucena, V., Raya-Trenas, A., Herruzo, J. y Herruzo, C. (2019). Addiction or abuse of ICTs? In: M. J. Osuna y M $\mathrm{M}^{\mathrm{a}} \mathrm{I}$. Amor (Eds.), La transversalidad de género (pp. 111-121). Madrid: Síntesis.

Miller, S. M. (2008) The effect of frequency and type of internet use on perceived social support and sense of well-being in individuals with spinal cord injury. Rehabilitation Counseling Bulletin, 51, 148-158.

Organización Mundial de la Salud (2019). International Classification of Diseases. Recuperado de https://icd. who.int/browse11/1-m/en.

Palmieri, G., Evans, C., Hansen, V., Brancaleoni, G., Ferrari, S., Porcelli, P., ... Rigatelli, M. (2009). Validation of the Italian version of the clinical outcomes in routine evaluation outcome measure (CORE-OM). Clinical Psychology and Psychotherapy, 16, 444-449. doi:10.1002/ cpp.646.

Perry, M., O'hara, K., Sellen, A., Brown, B. y Harper, R. (2001). Dealing with mobility: Understanding access anytime, anywhere. ACM Transactions on Computer-Human Interaction, 8, 323-347. doi:10.1145/504704.504707.

Pino, M. J., Herruzo, J., Raya, A., Ruiz-Olivares, R. y Herruzo, C. (2020). Development of IAT-12, a reduced Spanish version of the Internet Addiction Test. Current Psychology. doi:10.1007/s12144-020-01167-4.

Ruiz-Olivares, R., Lucena, V. Pino, M. J. y Herruzo, J. (2010). Análisis de comportamientos relacionados con el uso/abuso de internet, teléfono móvil, compras y juego en estudiantes universitarios. Adicciones, 22, 301-310. doi:10.20882/adicciones.171.

Simpson, C. G., Rose, C. A. y Ellis, S. K. (2016). Gender discrepancies and victimization of students with disa- bilities. Remedial and Special Education, 37, 296-307. doi:10.1177/0741932516646082.

Söderström, S. (2009). The digital town square: Information and communication technology as an opportunity and a barrier to social relations for disabled youth. In: C. A. Marshall, E. Kendall, M. E. Banks y R. M. S. Gover (Eds.), Disabilities: Insights from across fields and around the world, vol 2: The context: Environmental, social, and cultural considerations; disabilities: Insights from across fields and around the world (pp. 61-80, Chapter XXII, 331 Pages). Santa Barbara, CA: Praeger/ABC-CLIO.

Söderström, S. (2011). Staying safe while on the move: Exploring differences in disabled and non-disabled young people's perceptions of the mobile phone's significance in daily life. Young, 19, 91-109. doi:10.1177\% 2F110330881001900106.

Spada, M. M. (2014). An overview of problematic internet use. Addictive Behaviors, 39, 3-6. doi:10.1016/j.addbeh.2013.09.007.

Suriá, R. (2012a). La discapacidad en nuestros jóvenes, ¿fomenta las relaciones normalizadas a través de las redes sociales online o les hace adictos a ellas? Revista Electrónica de Tecnología Educativa, 41, 1-17.

Suriá, R. (2012b). Redes sociales online y su utilización para mejorar las habilidades sociales en jóvenes con discapacidad. Escritos de Psicología, 5, 16-23. doi:10.5231/ psy.writ.2012.1809.

Suriá, R. (2015). Disability in young people, increases the risk of excessive internet use? Health and Addictions, 15, $15-24$.

Trujillo, A., Feixas, G., Bados, A., García-Grau, E., Salla, M., Medina, J.... Evans, C. (2016). Psychometric properties of the Spanish version of the Clinical Outcomes in Routine Evaluation - Outcome Measure. Neuropsychiatric Disease and Treatment, 12, 1457. doi:10.2147/NDT.S103079.

Young, K. S. (1998). Internet addiction: The emergence of a new clinical disorder. Cyberpsychology and Behavior, 1, 237-244. doi:10.1089/cpb.1998.1.237.

Young, K. S. (2011). Clinical assessment of internet-addicted clients. In: K. Young y C. Abreu (Eds.), Internet addiction: A handbook and guide to evaluation and treatment (pp. 19-34). New Jersey: John Wiley \& Sons.

Zhou, Y., Li, D., Li, X., Wang, Y. y Zhao, L. (2017). Big five personality and adolescent internet addiction: The mediating role of coping style. Addictive Behaviors, 64, 42-48. doi:10.1016/j.addbeh.2016.08.009. 